\title{
Screening of Bufadienolides from Toad Venom Identifies Gammabufotalin as a Potential Anti-inflammatory Agent
}

Authors

Yuanru Zheng ${ }^{1,2 *}$, Lijuan Deng ${ }^{3 *}$, Huihui Cao ${ }^{1,2 *}$, Nishan Xu ${ }^{1,2}$, Dongmei Zhang ${ }^{4}$, Haiyan Tian ${ }^{4}$, Baojing Li ${ }^{5}, Z^{i b i n}$ Lu $^{1,2}$, Wencai Ye ${ }^{4}$, Linzhong Yu ${ }^{1,2}$, Chunlin Fan ${ }^{4}$, Junshan Liu ${ }^{1,2}$

\section{Affiliations}

1 Third Level Research Laboratory of State Administration of Traditional Chinese Medicine, School of Traditional Chinese Medicine, Southern Medical University, Guangzhou, P.R. China

2 Guangdong Provincial Key Laboratory of Chinese Medicine Pharmaceutics, Guangzhou, P. R. China

3 Formula-pattern Research Center, School of Traditional Chinese Medicine, Jinan University, Guangzhou, P. R. China

4 Guangdong Province Key Laboratory of Pharmacodynamic Constituents of TCM and New Drugs Research, College of Pharmacy, Jinan University, Guangzhou, P. R. China

5 College of Traditional Chinese Medicine, Yunnan University of Chinese Medicine, Kunming, P. R. China

\section{Key words}

bufadienolides, gammabufotalin, bufonidae, Bufo gargarizans, anti-inflammation, zebrafish, Danio rerio

$\begin{array}{ll}\text { received } & \text { February } 1,2020 \\ \text { accepted after revision } & \text { August 23, 2020 } \\ \text { published online } & \text { October } 13,2020\end{array}$

\section{Bibliography}

Planta Med 2022; 88: 43-52

DOI 10.1055/a-1248-2626

ISSN 0032-0943

(c) 2020. Thieme. All rights reserved.

Georg Thieme Verlag KG, Rüdigerstraße 14,

70469 Stuttgart, Germany

Correspondence

Associate Prof. Junshan Liu

School of Traditional Chinese Medicine,

Southern Medical University

No. 1838, North Guangzhou Avenue, Guangzhou 510515,

P. R. China

Phone: + 862061648539 , Fax: + 862061648539

liujunshan@smu.edu.cn
Correspondence

Associate Prof. Chunlin Fan

Guangdong Province Key Laboratory of Pharmacodynamic Constituents of TCM and New Drugs Research, College of Pharmacy, Jinan University

No. 601, West Huangpu Avenue, Guangzhou 510632,

P. R. China

Phone: +862085222653 , Fax: +862085222653

jnuchunlin@163.com

Correspondence

Prof. Linzhong Yu

School of Traditional Chinese Medicine,

Southern Medical University

No. 1838, North Guangzhou Avenue, Guangzhou 510515, P.R. China

Phone: + 862061648262 , Fax: +862061648262

yulzh@smu.edu.cn

\begin{abstract}
Toad venom (Chansu) is used in the treatment of infectious and inflammatory diseases in China and East/Southeast Asian countries. However, the anti-inflammatory components of toad venom have not yet been systematically evaluated and clearly defined. To investigate the anti-inflammatory effects of toad venom and identify new anti-inflammatory ingredients, we used zebrafish, an alternative drug screening model, to evaluate the anti-inflammatory effects of 14 bufadienolides previously isolated from toad venom. Most of the bufadienolides were found to exert significant anti-inflammatory effects on lipopolysaccharide-, $\mathrm{CuSO}_{4}$, , or tail transection-induced zebrafish inflammatory models. Moreover, gammabufotalin (6) inhibits lipopolysaccharide-induced inflammation by suppressing the myeloid differentiation primary response 88/nuclear factor-kappa B and STAT3 signal pathways. This study confirms the potential of zebrafish in drug screening, clarifies the anti-inflammatory effects of bufadienolides from toad venom, and indicates that gammabufotalin may be developed as a novel therapeutic agent for inflammatory diseases in the future.
\end{abstract}

* These authors contributed equally to this work. 


$\begin{array}{ll}\text { ABBREVIATIONS } \\ \text { ANOVA } & \text { analysis of variance } \\ \text { COX-2 } & \text { cyclooxygenase-2 } \\ \text { dpf } & \text { days post-fertilization } \\ \text { GFP } & \text { green fluorescent protein } \\ \text { H\&E } & \text { hematoxylin and eosin } \\ \text { IKB } \alpha & \text { inhibitor of kappa B } \\ \text { IL } & \text { interleukin } \\ \text { LPS } & \text { lipopolysaccharide } \\ \text { mpo } & \text { myeloperoxidase } \\ \text { MyD88 } & \text { myeloid differentiation primary response } 88 \\ \text { NF- } \text { B } & \text { nuclear factor-kappa B } \\ \text { qRT-PCR } & \text { quantitative real-time polymerase chain reaction } \\ \text { STAT3 } & \text { signal transducer and activator of transcription } 3\end{array}$

\section{Introduction}

Toad venom (Chansu) is a traditional Chinese medicine that consists of the self-defensive secretions from the auricular and skin glands of the Asiatic toad Bufo gargarizans or the Asian common toad Duttaphrynus melanostictus (synonym Bufo melanostictus) $[1,2]$. It is widely used in China alone or combined with other drugs in the treatment of respiratory infections and inflammatory diseases, such as hepatitis, otitis media, tonsillitis, pulpitis, pericoronitis, and arthritis $[3,4]$. Toad venom is usually processed by drying and pulverizing in order to be used in pills or topical formulations [5]. Bufadienolides are the main bioactive components of toad venom. Several studies have reported the anti-inflammatory activities of bufadienolides, especially on cancer-related inflammation [6,7]. Cinobufagin inhibited the NF- $\kappa B$ signaling pathway and decreased the production of TNF- $\alpha, \mathrm{IL}-1 \beta$, and IL-12 in LPSstimulated dendritic cells [8]. Bufalin attenuated the protein expression levels of the proinflammatory mediators TNF- $\alpha$, IL-6, COX-2, and IL- $1 \beta$ through NF- $\kappa$ B signaling in carrageenan-induced paw edema in rats [6]. These findings highlight that toad venom has a promising future as an anti-inflammatory drug. However, the anti-inflammatory components of toad venom have not yet been systematically evaluated and clearly defined.

Zebrafish has emerged as a novel and alternative model for high-throughput screening for drug discovery $[9,10]$. Compared to cell lines, a zebrafish possesses fully developed vertebrate organ systems, and phenotype-based screening using a zebrafish model enables the study of a much broader range of biological processes [9]. Compared to murine models, experiments on zebrafish are easier to perform [11]. More importantly, accumulated evidence indicates that the immune system of the zebrafish is highly conserved with that of humans, including multiple immune cells (neutrophils, macrophages, and lymphocytes) and a large number of inflammatory genes (IL-6, TNF- $\alpha$, NF- $K B$, etc.) [12-14].

MyD88, a critical adapter protein for toll-like receptor 4 , leads to the activation of downstream NF- $k \mathrm{~B}$ and the subsequent production of proinflammatory cytokines, such as TNF- $\alpha$, IL-6, COX2 , and IL-1 $\beta$ [15]. STAT3 also plays a pivotal role in mediating the cascade response of inflammation [16]. In the current study, zebrafish inflammatory models were established to evaluate the anti-inflammatory effects of 14 bufadienolides previously isolated from toad venom [17] and the inhibitory effects of a selected bufadienolide, gammabufotalin, on proinflammatory mediators. The MyD88/NF- $к B$ and STAT3 pathways were also examined.

\section{Results}

The structures of the bufadienolides isolated from toad venom are shown in - Fig. 1. To identify their in vivo anti-inflammatory activities, a $T g$ (mpo-GFP) transgenic zebrafish line was used. In this zebrafish, mpo, tagged with GFP, is expressed in neutrophils, which makes it possible to track neutrophil behavior in vivo during the inflammatory process [18].

LPS is a typical proinflammatory agent that can trigger an inflammatory response and consequently activate a series of signaling cascades. Our research group initiatively established a zebrafish inflammatory model by microinjecting LPS into the yolk [19], which has been widely used in the rapid screening of anti-inflammatory drugs in vivo [20-22]. In this study, the anti-inflammatory effects of 14 bufadienolides at nontoxic doses $(1,4$, and 12: $50 \mu \mathrm{M} ; 2$, 13, and 14: $10 \mu \mathrm{M} ; 3$ and 6: $5 \mu \mathrm{M} ; 5$ and 8: $1 \mu \mathrm{M} ; 7,9$, and 11: $0.5 \mu \mathrm{M} ; 10: 4 \mu \mathrm{M})$ were evaluated for the first time using an LPS-stimulated zebrafish inflammatory model. As shown in - Fig. 2, a large number of neutrophils can be found in the yolks after LPS microinjection, while bufadienolides or dexamethasone (positive control, $5 \mu \mathrm{g} / \mathrm{mL}$ ) reduced the recruitment of neutrophils in the yolk sac. Gammabufotalin (6), bufalin (8), and 19-oxodesactelylcinobutofalin (12) showed the most remarkable inhibitory effects.

$\mathrm{CuSO}_{4}$ and tail transection can injure the lateral line neuromast areas or tails of zebrafish, and these two inflammatory models have been used for large-scale chemical screenings to seek new effective compounds [23-25]. Thus, the effects of 14 bufadienolides were further confirmed in $\mathrm{CuSO}_{4}$ - or tail transection-induced inflammatory models. Our results showed that the accumulation of neutrophils was significantly reduced in the injured areas after treatment with bufadienolides, except for 1, 2 and 5 ( $\triangleright$ Figs. 3 and 4), which confirms that most bufadienolides have strong anti-inflammatory activities, especially 6 and 12 .

To further identify the anti-inflammatory activities of bufadienolides and explore their underlying mechanisms, gammabufotalin (6) was chosen for the next experiments.

Survival rate analysis was carried out to visually measure the defensive efficacy of zebrafish on LPS. As shown in > Fig. 5, all zebrafish died within $68 \mathrm{~h}$ post-injection in the LPS group, whereas more than $40 \%$ of zebrafish survived in gammabufotalin group with the same treatment duration, which indicates that gammabufotalin exhibits protective effects on zebrafish challenged by LPS.

Infiltration of inflammatory cells is a critical process during inflammation [26]. We observed the histopathological features of LPS-infected zebrafish by H\&E staining. As shown in > Fig. 6, infiltration of inflammatory cells was clearly observed in LPS-induced zebrafish. As expected, the histopathological features were ameliorated by gammabufotalin treatment, which further confirms that gammabufotalin exerts anti-inflammatory effects in LPS-microinjected zebrafish. 


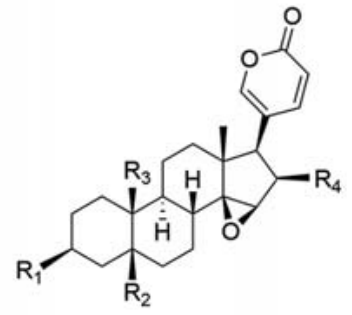

a: $\sum_{0} \prod_{0}^{\mathrm{OCH}_{3}}$

\begin{tabular}{|c|c|c|c|c|c|}
\hline \multirow{2}{*}{ No. } & \multirow{2}{*}{ Compound } & \multicolumn{4}{|c|}{ Substitutional group } \\
\hline & & $R_{1}$ & $\mathrm{R}_{2}$ & $\mathrm{R}_{3}$ & $\mathrm{R}_{4}$ \\
\hline 1 & cinobufagin-3-hemisuberate methyl ester & a & $\mathrm{H}$ & $\mathrm{CH}_{3}$ & OAC \\
\hline 4 & $\begin{array}{l}(3 \beta, 5 \beta, 15 \beta, 16 \beta)-14,15 \text {-epoxy-3,5,10,16- } \\
\text { tetrahydroxy-19-norbufa-20,22-dienolide }\end{array}$ & $\mathrm{OH}$ & $\mathrm{OH}$ & $\mathrm{OH}$ & $\mathrm{OH}$ \\
\hline 11 & $\begin{array}{l}16 \beta \text {-acetoxy-14,15 } \beta \text {-epoxy- } 3 \beta, 5,19 \text {-trihydroxy- } 5 \beta \text {-bufa- } \\
20,22 \text {-dienolide }\end{array}$ & $\mathrm{OH}$ & $\mathrm{OH}$ & $\mathrm{CH}_{2} \mathrm{OH}$ & OAC \\
\hline 12 & 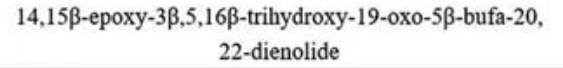 & $\mathrm{OH}$ & $\mathrm{OH}$ & $\mathrm{CHO}$ & $\mathrm{OH}$ \\
\hline
\end{tabular}

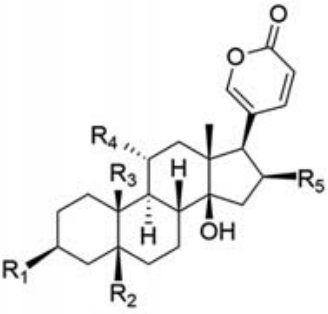<smiles>N=C(N)NCCCC(NC(=O)CCCCCCC(=O)O[14C](=[18O])[18F])C(=O)O</smiles>

\begin{tabular}{ccccccc}
\hline \multirow{2}{*}{ No. } & \multirow{2}{*}{ Compound } & \multicolumn{5}{c}{ Substitutional group } \\
\cline { 3 - 7 } & & $\mathrm{R}_{1}$ & $\mathrm{R}_{2}$ & $\mathrm{R}_{3}$ & $\mathrm{R}_{4}$ & $\mathrm{R}_{5}$ \\
\hline $\mathbf{3}$ & bufalin-3-suberylarginate & $\mathbf{b}$ & $\mathrm{H}$ & $\mathrm{CH}_{3}$ & $\mathrm{H}$ & $\mathrm{H}$ \\
$\mathbf{5}$ & hellebrigenin & $\mathrm{OH}$ & $\mathrm{OH}$ & $\mathrm{CHO}$ & $\mathrm{H}$ & $\mathrm{H}$ \\
$\mathbf{6}$ & gammabufotalin & $\mathrm{OH}$ & $\mathrm{H}$ & $\mathrm{CH}_{3}$ & $\mathrm{OH}$ & $\mathrm{H}$ \\
$\mathbf{8}$ & bufalin & $\mathrm{OH}$ & $\mathrm{H}$ & $\mathrm{CH}_{3}$ & $\mathrm{H}$ & $\mathrm{H}$ \\
$\mathbf{9}$ & 19-hydroxybufalin & $\mathrm{OH}$ & $\mathrm{H}$ & $\mathrm{CH}_{2} \mathrm{OH}$ & $\mathrm{H}$ & $\mathrm{H}$ \\
$\mathbf{1 0}$ & bufotalin & $\mathrm{OH}$ & $\mathrm{H}$ & $\mathrm{CH}_{3}$ & $\mathrm{H}$ & $\mathrm{OAc}$ \\
$\mathbf{1 3}$ & telocinobufagin & $\mathrm{OH}$ & $\mathrm{OH}$ & $\mathrm{CH}_{3}$ & $\mathrm{H}$ & $\mathrm{H}$ \\
$\mathbf{1 4}$ & hellebrigenol & $\mathrm{OH}$ & $\mathrm{OH}$ & $\mathrm{CH}_{2} \mathrm{OH}$ & $\mathrm{H}$ & $\mathrm{H}$ \\
\hline
\end{tabular}

b

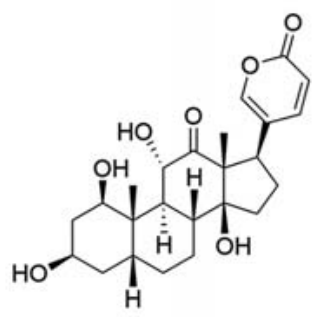

2

c

(1 $\beta$-hydroxyarenobufagin)

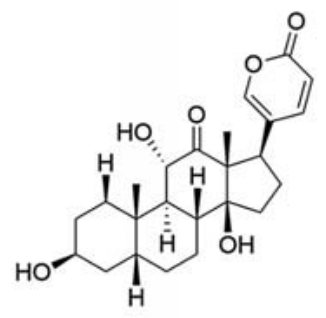

7

(arenobufagin)

- Fig. 1 Chemical structures of bufadienolides isolated from toad venom.

The proinflammatory cytokine mediators IL- 6 and TNF- $\alpha$ play important roles in inflammation $[27,28]$ and can be overexpressed after LPS stimulation [29]. To investigate whether gammabufotalin regulates the expression levels of IL- 6 and TNF- $\alpha$, qRT-PCR analysis was employed to evaluate the mRNA levels of IL-6 and TNF- $\alpha$ in LPS-infected zebrafish. As shown in > Fig. 7,
IL-6 and TNF- $\alpha$ mRNA levels were increased remarkably in LPS-microinjected zebrafish. Gammabufotalin significantly inhibited LPSinduced IL- 6 and TNF- $\alpha$ upregulation, suggesting that gammabufotalin exerts its anti-inflammatory effects by inhibiting the expression levels of proinflammatory cytokines in vivo. 


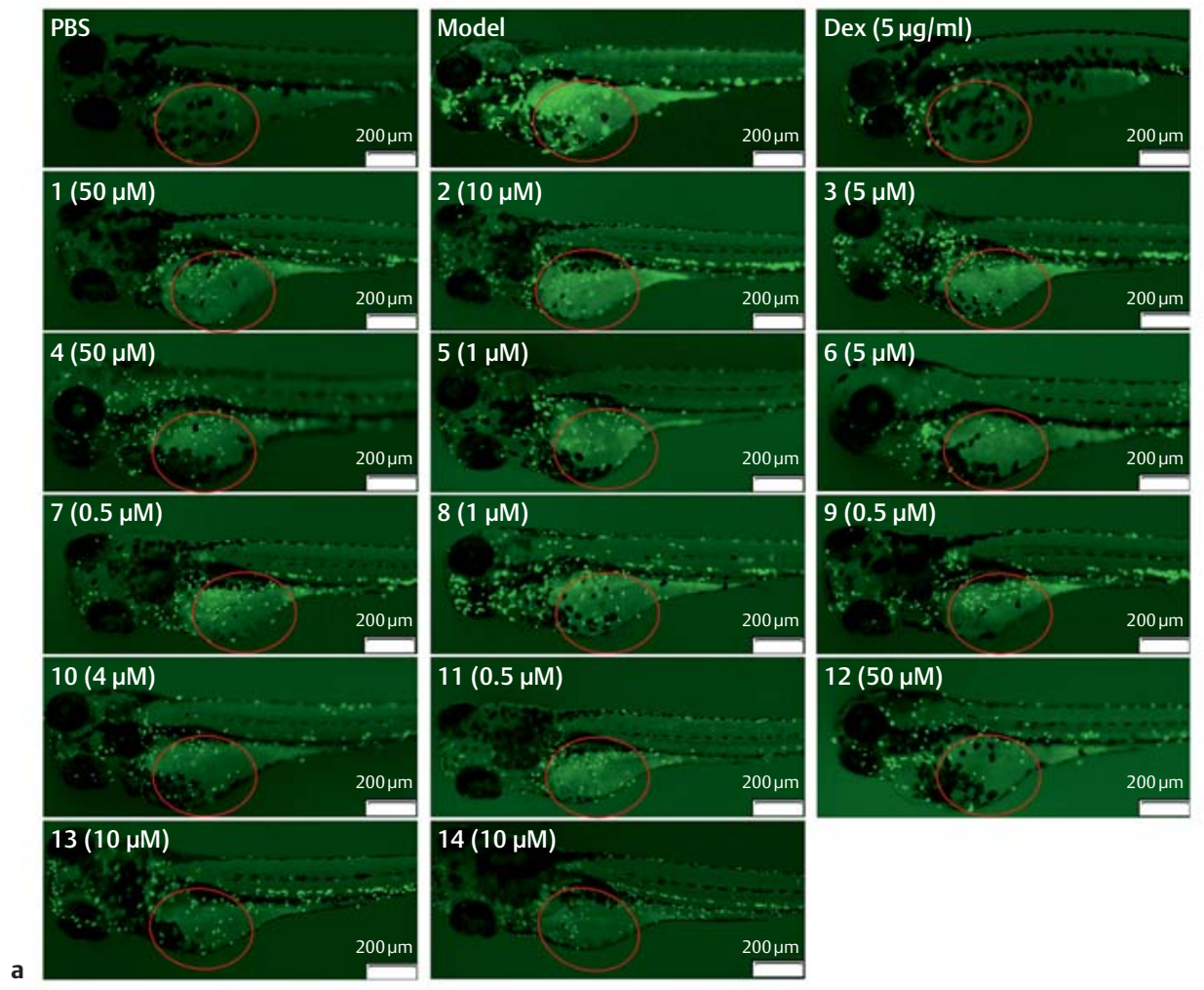

b

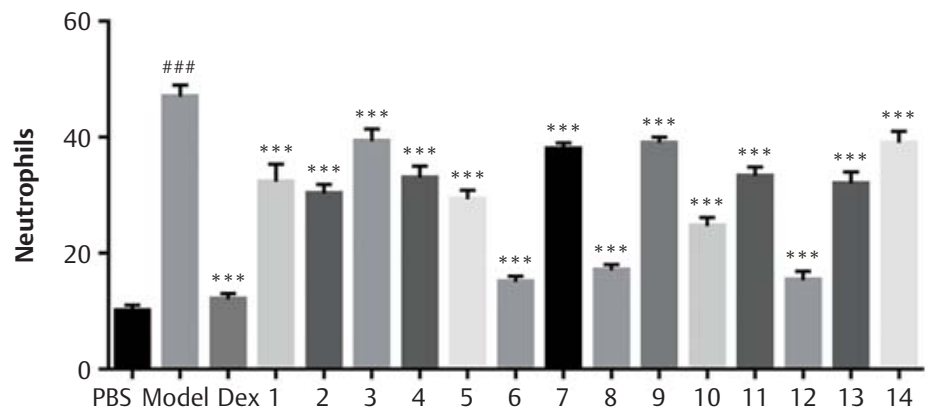

- Fig. 2 Bufadienolides reduce the accumulation of neutrophils in 3-dpf Tg(mpo:GFP) larvae subjected to LPS. a Representative images of zebrafish in the LPS-induced inflammatory model (scale bar $=200 \mu \mathrm{m}$ ). Dexamethasone (Dex) was utilized as the positive control. b The number of neutrophils in the region of interest (red circles) were counted. Data are represented as the mean \pm SD of three independent experiments $(n=20)$.

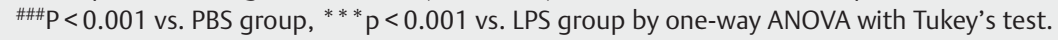

The NF- $\kappa B$ signaling pathway plays a vital role during the process of inflammation and can be activated by MyD88 in response to the LPS stimulus [30]. The phosphorylation and ubiquitylation of $I_{\kappa} \mathrm{B} \alpha$ can promote the release and translocation of NF- $\kappa \mathrm{B}$ into the nucleus, triggering the secretion of multiple inflammatory cytokines [31]. STAT3 is another important transcription factor in inflammation that can be activated by LPS and then translocate into the nucleus to regulate specific genes [16]. We thus examined the mRNA expression levels of NF- $\kappa B, I_{k B} \alpha$, MyD88, and STAT3 to further elucidate the action mechanisms of gammabufotalin. Our results showed that gammabufotalin remarkably inhibited the upregulation of NF- $\kappa \mathrm{B}, \mathrm{I}_{\kappa} \mathrm{B} \alpha, \mathrm{MyD} 88$, and STAT3 in LPS-stimulated zebrafish ( $\vee$ Fig. 8), suggesting that the anti-inflammatory effects of gammabufotalin are closely associated with inhibition of MyD88/NF- $\kappa$ B and STAT3 signal pathways (• Fig. 9).

\section{Discussion}

In recent years, the zebrafish has become a prominent vertebrate model in biomedical research. Besides the advantages of small size, rapid growth, high productive rate, relatively transparent embryos, and amenability to genetic manipulation, the zebrafish has high similarities with humans in disease type and innate immune system [32]. Zebrafish embryos or larvae facilitate highthroughput in vivo experiments, and a large-scale screening on zebrafish has been performed for drug discovery [9]. In the present 


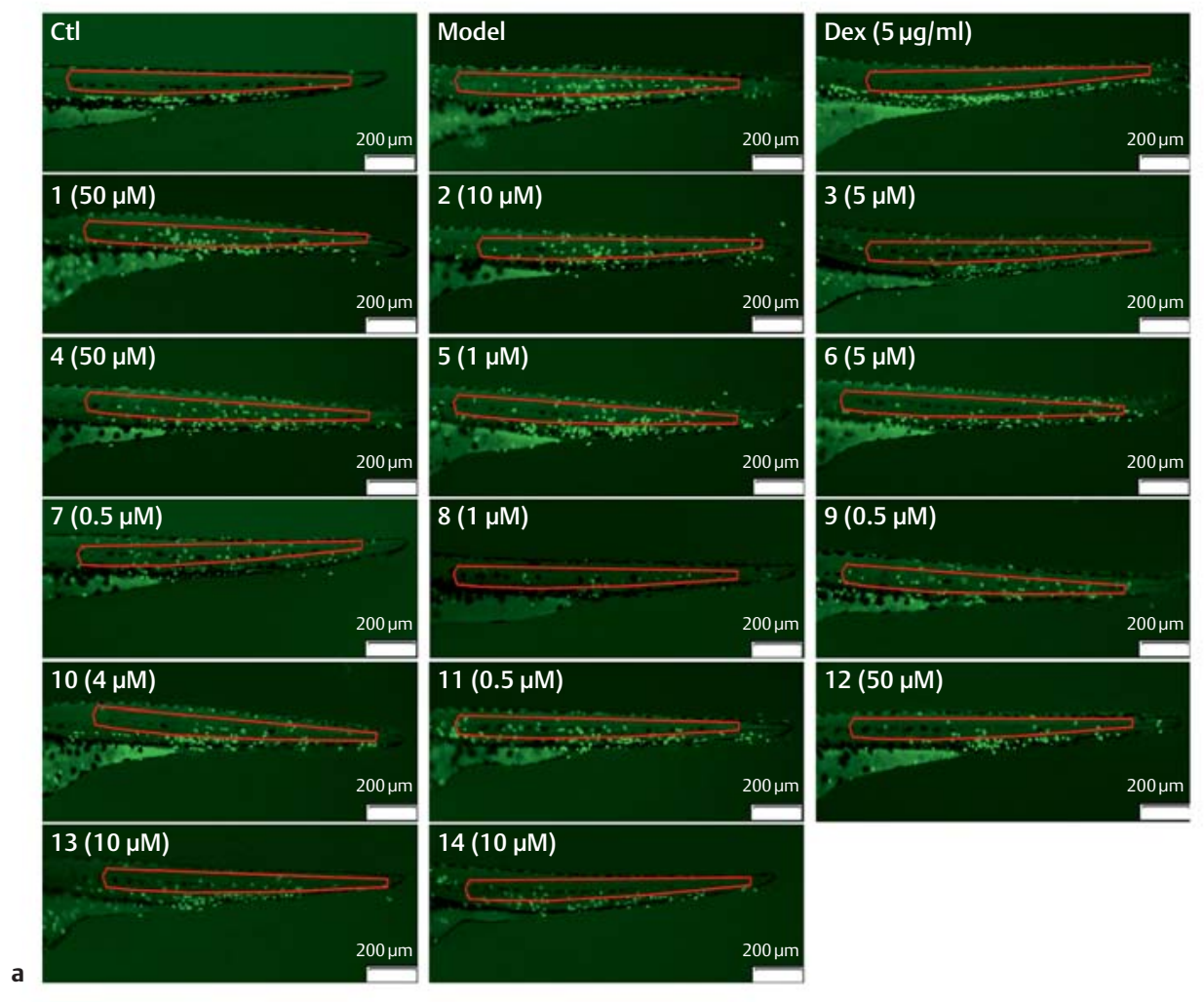

b

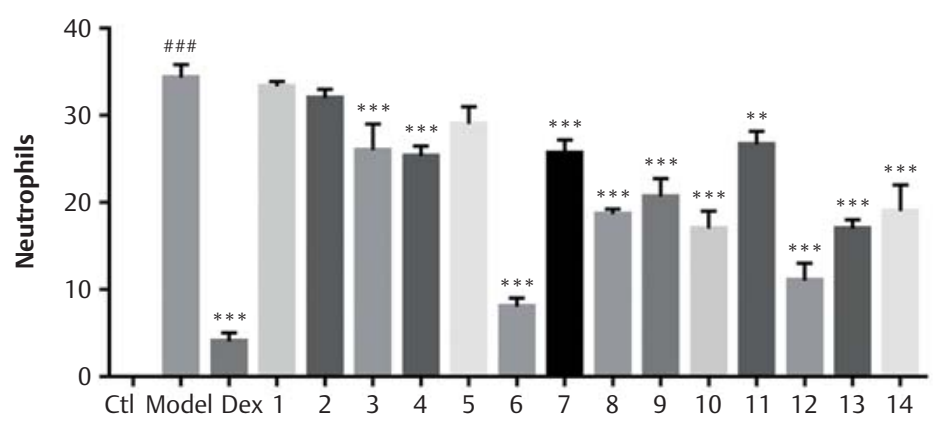

- Fig. 3 Bufadienolides, except for 1, 2, and 5, reduce the accumulation of neutrophils in 3-dpf Tg(mpo:GFP) larvae subjected to CuSO $\mathrm{S}_{4}$ a Representative images of zebrafish in the $\mathrm{CuSO}_{4}$-induced inflammatory model (scale bar $=200 \mu \mathrm{m}$ ). Dexamethasone (Dex) was utilized as the positive control. $\mathbf{b}$ The number of neutrophils in the region of interest (red irregular rectangles) were counted. Data are represented as the mean \pm SD of three independent experiments $(n=15)$. ${ }^{\# \#} \mathrm{P}<0.001$ vs. control group, ${ }^{* * *} \mathrm{p}<0.001 \mathrm{vs}$. model group by one-way ANOVA with Tukey's test.

study, transgenic line $\mathrm{Tg}(\mathrm{mpo}:$ GFP), a neutrophil-labeled zebrafish line, was used. Neutrophils are major effectors of acute inflammation and innate immune responses. They can be recruited to infected or injured tissues by proinflammatory cytokines such as TNF- $\alpha$ and IL-6. Deregulation of neutrophils and their hyperactivity can lead to tissue damage in severe inflammation or trauma [33]. Neutrophil-labeled zebrafish allow us to observe the biological behavior of larvae neutrophils in real time, which facilitates anti-inflammatory drugs screening. LPS-, $\mathrm{CuSO}_{4}$, and tail transection-induced zebrafish inflammatory models were established in our study. They respectively represent inflammations that are caused by bacterial infection and chemical and physical injuries. Our research group has successfully used these models in studying traditional Chinese medicines on inflammatory diseases [20,34].

Toad venom has been traditionally used for treating infectious and inflammatory diseases in China for thousands of years. In the Chinese Pharmacopoeia, toad venom is considered a therapeutic agent for a carbuncle abscess, swollen sore throat, diarrhea with abdominal pain, and so on, with detoxicant, anti-inflammatory, and antalgic effects [35]. Bufadienolides are considered the major bioactive components of toad venom. However, except for bufalin and cinobufagin [36], there is little known about the biological effects of bufadienolides. In the present study, we evaluated the anti-inflammatory activities of 14 bufadienolides from toad venom in 3 zebrafish inflammatory models. Our data showed that 


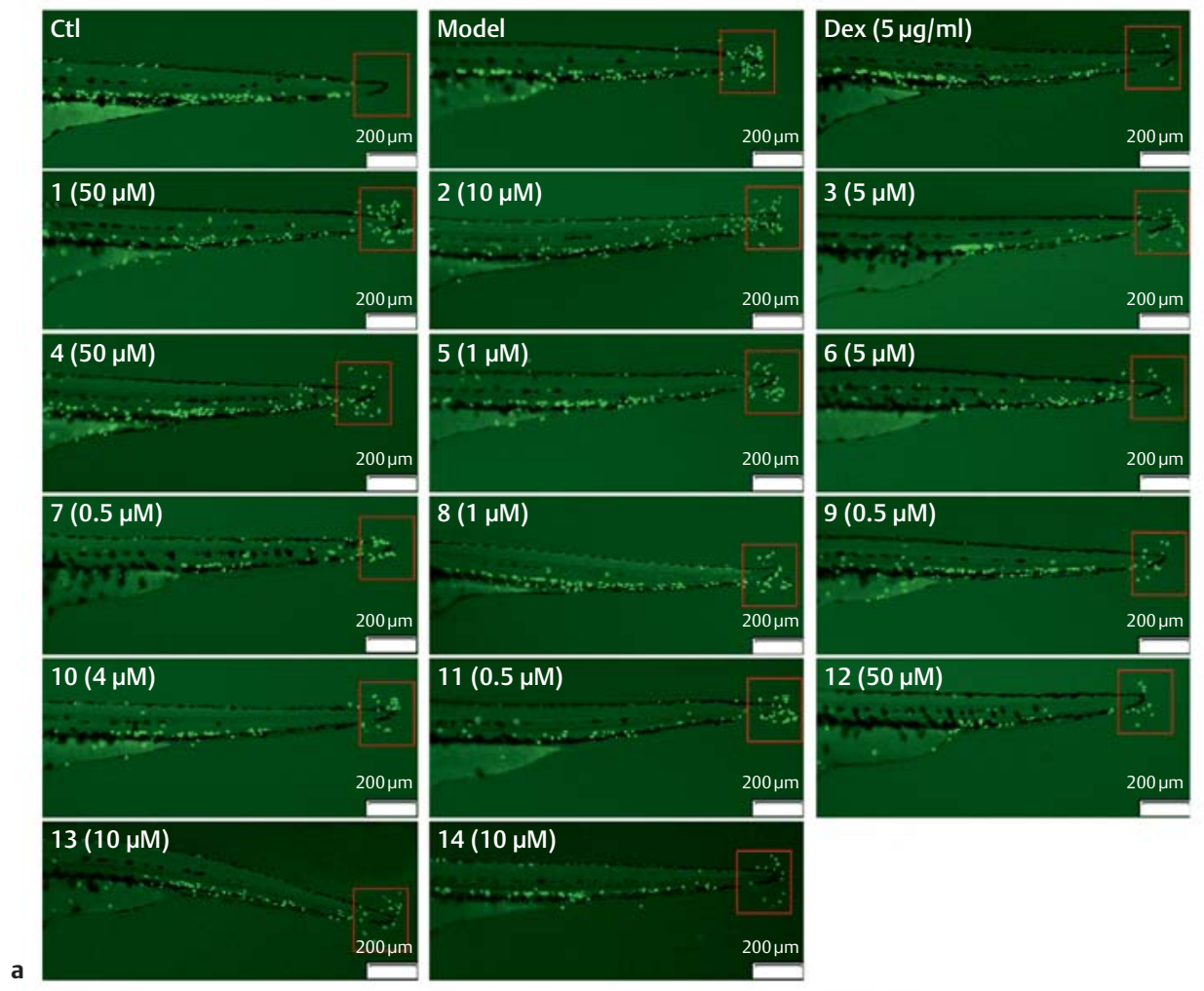

b

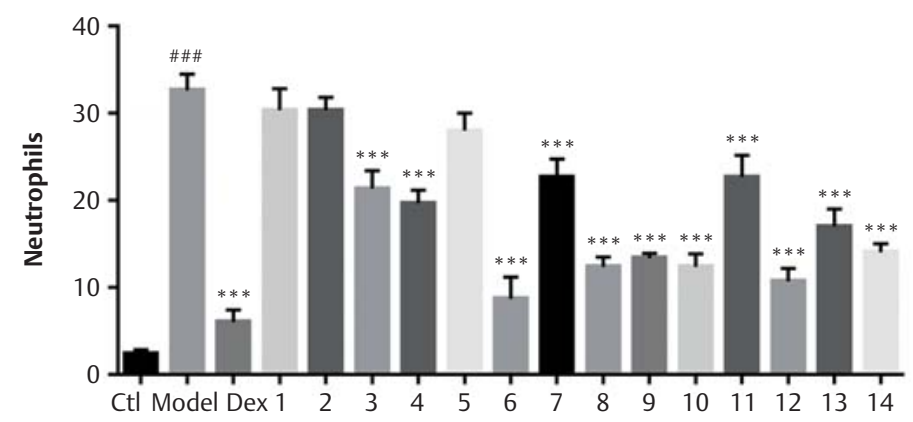

- Fig. 4 Bufadienolides, except for 1, 2, and 5, reduce the accumulation of neutrophils in 3-dpf Tg(mpo:GFP) larvae subjected to tail transection. a Representative images of zebrafish in the tail transection-induced inflammatory model (scale bar $=200 \mu \mathrm{m}$ ). Dexamethasone (Dex) was utilized as the positive control. $\mathbf{b}$ The number of neutrophils in the region of interest (red squares) were counted. Data are represented as the mean \pm SD of three independent experiments $(\mathrm{n}=15)$. ${ }^{\# \# P} \mathrm{P}<0.001$ vs. control group, ${ }^{* * *} \mathrm{p}<0.001$ vs. model group by one-way ANOVA with Tukey's test.

these bufadienolides, except for 1,2 , and 5 , have significant antiinflammatory effects, which were indicated by reduced migration and recruitment of neutrophils to the injured sites. However, the inhibitory activities of these compounds were slightly different in the three zebrafish models, which may be attributed to the different inflammatory agents, inflammatory sites, or action time of bufadienolides. Gammabufotalin possessed the most significant anti-inflammatory activity among these bufadienolides. The antiinflammatory properties of gammabufotalin were further confirmed by its effects on protecting zebrafish against LPS-induced death, alleviating inflammatory cell infiltration, and downregulating proinflammatory cytokines IL-6 and TNF- $\alpha$. According to the results from all three zebrafish inflammatory models, 12 showed more potent effects than 4 , suggesting that the introduction of an aldehyde group might be favorable to the anti-inflammatory activity. The difference of anti-inflammatory activities between 8 and 9 revealed that the presence of a hydroxyl group was unfavorable to the activity.

MyD88/NF-kB and STAT3 pathways that have been identified in zebrafish are the crucial hallmarks of inflammation and cancer $[37,38]$. On one hand, activated MyD88/NF-kB signaling can secrete cytokine IL-6 to directly stimulate the STAT3 pathway [39]. On the other hand, STAT3 can further enhance NF-kB activity via prolonging its nuclear retention. These data suggest that NF- $\kappa \mathrm{B}$ 


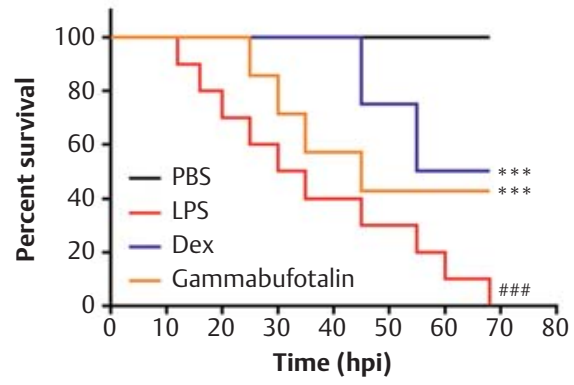

- Fig. 5 Protective effect of gammabufotalin (6) in LPS-induced 3-dpf $T g$ (mpo:GFP) larvae. Each survival curve represents data pooled from three independent experiments $(n=30)$. Dexamethasone (Dex) was utilized as the positive control. ${ }^{\# \# P<0.001 ~ v s . ~ P B S ~}$ group, ${ }^{* * *} \mathrm{p}<0.001$ vs. LPS group by one-way ANOVA with Tukey's test.

and STAT3 pathways are synergistically interacted in the inflammatory response [40]. We found that gammabufotalin significantly inhibited the mRNA levels of NF- $\kappa \mathrm{B}, \mathrm{I}_{\kappa} \mathrm{B} \alpha$, MyD88, and STAT3 in LPS-infected zebrafish larvae. These results indicate that the anti-inflammatory activity of gammabufotalin may be due to its inhibitory effects on MyD88/NF- $\mathrm{BB}$ and STAT3 signaling pathways.

In summary, our study shows for the first time that bufadienolides from toad venom possess anti-inflammatory activities in zebrafish inflammatory models. Gammabufotalin (6) exerts the most potent inhibitory effects, which are related to the MyD88/ $\mathrm{NF}-\kappa \mathrm{B}$ and STAT3 signaling pathways inhibition.

\section{Materials and Methods}

\section{Material and reagents}

The venom of $B$. gargarizans was bought from Baoyin Toad Breeding Base (Jiangsu, China). Bufadienolides were isolated and identified as previously reported [17]. Purity determined by HPLC was $\geq 95 \%$. The chromatographic separation was performed on a COSMOSIL- $\mathrm{C}_{18}$ column $(250 \mathrm{~mm} \times 4.6 \mathrm{~mm}, 5 \mu \mathrm{m})$ at $30^{\circ} \mathrm{C}$ using $\mathrm{MeOH}$ and $\mathrm{H}_{2} \mathrm{O}$ as the mobile phase at a flow rate of $1.0 \mathrm{~mL} / \mathrm{min}$. The chromatogram was monitored at $296 \mathrm{~nm}$. RNAiso Plus and an RT-PCR kit were provided by Takara. Dexamethasone was purchased from Guangzhou Baiyunshan Tianxin Pharmaceutical Co. Methylene blue was from Dalian Meilun Biotechnology Co and tricaine was from Shanghai Macklin Biochemical Co. DMSO, LPS, Escherichia coli 055: B5, and other reagents were obtained from Sigma-Aldrich.

\section{Zebrafish maintenance and embryos collection}

Tg(mpo:GFP) [18] zebrafish were a kind gift from Professor Wenqing Zhang of South China University of Technology. They were maintained under a 14-h light/10-h dark cycle in a recirculating aquatic habitat system ( $\mathrm{pH} 7.2-7.6$, salinity $0.03-0.04 \%)$ following standard guidelines for maintenance protocols [41].

Adult zebrafish were housed in a breeding tank in a male-to-female ratio of $1: 2$ to collect a sufficient number of embryos, which were subsequently transferred to a clean petri dish filled with egg water containing $0.002 \%$ methylene blue as a fungicide. Finally, these embryos were kept in a warm oven at $28.5^{\circ} \mathrm{C}$ for the following experiments.

\section{Determination of nontoxic concentrations of bufadienolides in zebrafish}

Larvae at $3 \mathrm{dpf}$ were divided into 24-well plates $(\mathrm{n}=20)$ and then treated with different concentrations of bufadienolides. Untreated zebrafish larvae served as the control group. After $24 \mathrm{~h}$, the dead embryos were recorded and the concentrations that caused a survival rate over $95 \%$ were used as the nontoxic concentrations.

\section{Lipopolysaccharide-induced inflammation model}

Larvae at $3 \mathrm{dpf}$ were anesthetized with $0.02 \%$ tricaine and immobilized in a clear petri dish coated with $2 \%$ agarose (Sigma-Aldrich). Subsequently $2 \mathrm{~nL}$ of LPS $(0.5 \mathrm{mg} / \mathrm{mL})$ were microinjected into the yolks to construct the inflammatory model $[19,20]$ by a cell microinjector (PM1000; MicroData Instrument, Inc.).

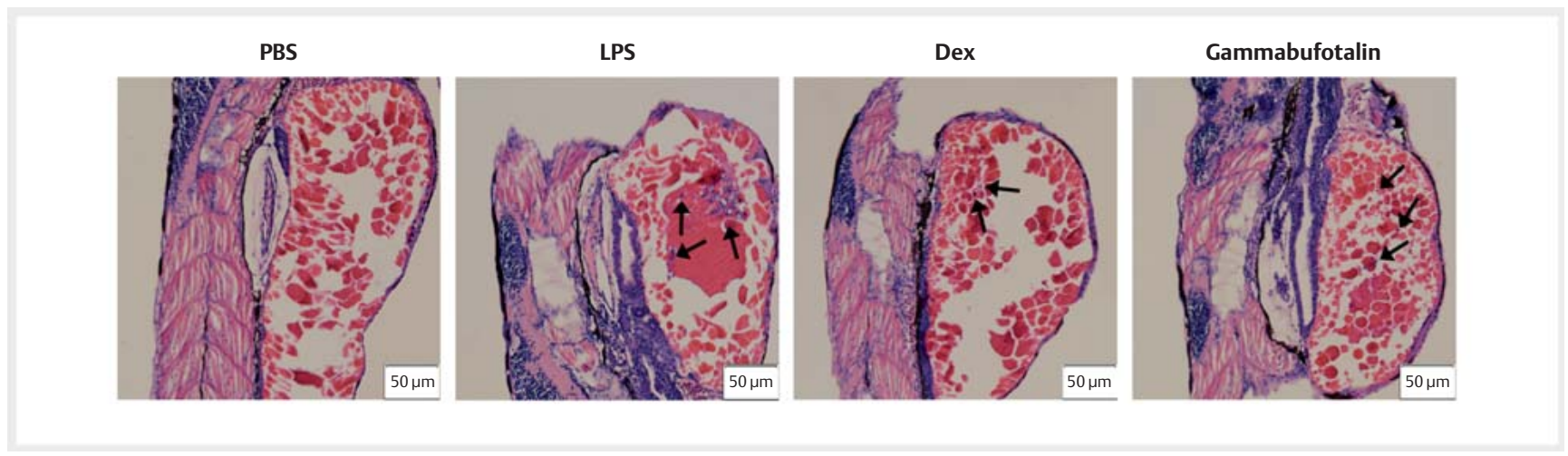

- Fig. 6 Gammabufotalin alleviates the inflammatory cell infiltration in LPS-induced 3-dpf Tg(mpo:GFP) larvae. The zebrafish larvae were microinjected with LPS in the presence or absence of gamabutofalin. Dexamethasone (Dex) was utilized as the positive control. After dehydration and embedding, paraffin sections $(4 \mu \mathrm{m})$ were stained by H\&E. The black arrows represent the inflammatory cells (scale bar $=50 \mu \mathrm{m}, \mathrm{n}=30$ ). 

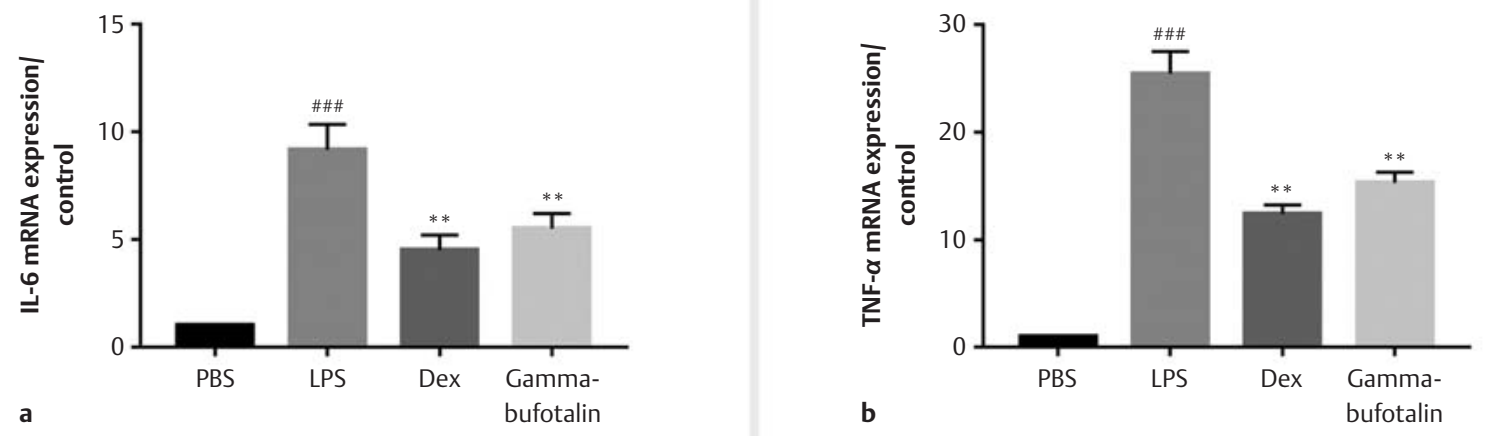

- Fig. 7 Gammabufotalin reduces the mRNA expression levels of IL-6 and TNF- $\alpha$ in LPS-induced 3-dpf Tg(mpo:GFP) larvae. a The mRNA expression level of IL-6. $b$ The mRNA expression level of TNF- $\alpha$. Data are represented as the mean \pm SD of three independent experiments $(n=30)$. Dexamethasone (Dex) was utilized as the positive control. ${ }^{\# \#} \mathrm{P}<0.001$ vs. PBS groups, ${ }^{*} p<0.01$ vs. LPS groups by one-way ANOVA with Tukey's test.

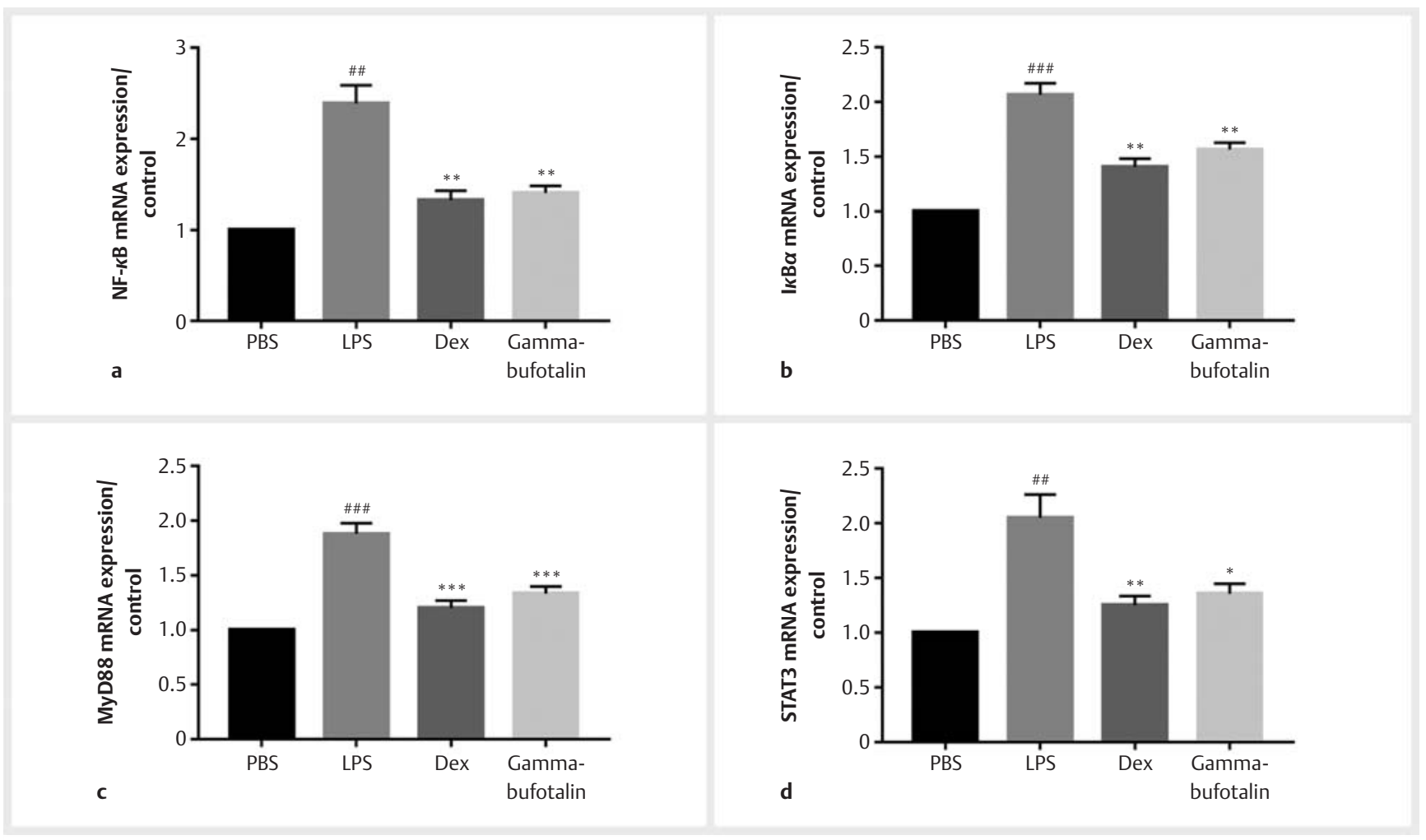

- Fig. 8 The effects of gammabufotalin on the mRNA expression levels of NF- $\kappa B$, IKB $\alpha$, MyD88, and STAT3 in LPS-induced 3-dpf Tg(mpo: GFP) larvae. a The mRNA expression level of NF- $\kappa B$. $b$ The mRNA expression level of $1 \kappa B \alpha$. $\mathbf{c}$ The mRNA expression level of MyD88. $\mathbf{d}$ The mRNA expression level of STAT3. Data are represented as the mean \pm SD of three independent experiments $(n=30)$. Dexamethasone (Dex) was utilized as the positive control. ${ }^{\# \#} \mathrm{P}<0.01,{ }^{\# \# \#} \mathrm{p}<0.001$ vs. PBS group, ${ }^{*} \mathrm{p}<0.05,{ }^{* *} \mathrm{p}<0.01,{ }^{* * *} \mathrm{p}<0.001$ vs. LPS group by one-way ANOVA with Tukey's test.

Next, these larvae were divided randomly into a 24-well plate ( $n=20 /$ well) and treated with various bufadienolides. Dexamethasone $(5 \mu \mathrm{g} / \mathrm{mL})$ served as a positive control and PBS was used as the vehicle control. Behavioral assessment of neutrophils was performed at $12 \mathrm{~h}$ post-LPS microinjection using a fluorescence microscope (MVX10; Olympus).

\section{$\mathrm{CuSO}_{4}$-induced inflammation model}

Larvae at $3 \mathrm{dpf}$ were randomly placed in a 24-well plate with 15 zebrafish/well and exposed to $\mathrm{CuSO}_{4}(20 \mu \mathrm{M})$ in the presence or absence of various bufadienolides for $2 \mathrm{~h}$. Untreated zebrafish larvae were used as the control group. Dexamethasone $(5.0 \mu \mathrm{g} / \mathrm{mL})$ was used as the positive control and the images were captured to evaluate the recruitment of neutrophils. 


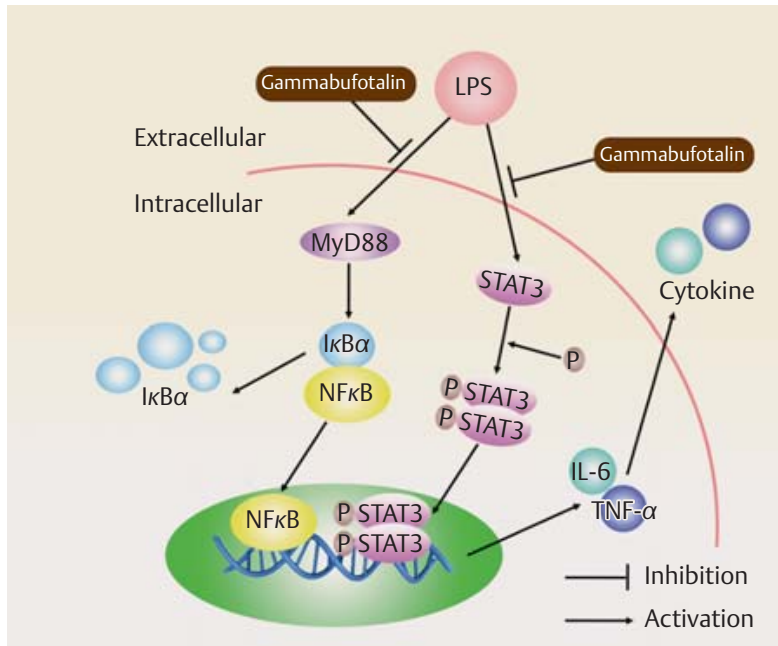

- Fig. 9 Possible anti-inflammatory mechanisms of gammabufotalin.

\section{Tail transection-induced inflammation model}

Larvae were injured at $3 \mathrm{dpf}$ by transection of the caudal fin with a scalpel blade under a stereomicroscope (Olympus; SZX7). Next, these injured larvae were incubated with various bufadienolides for $6 \mathrm{~h}$, mounted in $2 \%$ agarose, and imaged to observe the behavior of the fluorescent neutrophils. Untreated zebrafish larvae were used as the control group $(n=15)$.

\section{Survival analysis}

After microinjection, deceased zebrafish were counted every day and this experiment lasted for 3 days $(n=30)$ [19-21].

\section{Histopathological analysis}

Twelve hours after LPS microinjection with or without drug administration, the zebrafish $(n=30)$ were fixed in $4 \%(w / v)$ paraformaldehyde, dehydrated in graded ethanol, embedded with paraffin (Leica), and cut into $4-\mu \mathrm{m}$ sections. The specimens were subsequently stained with $\mathrm{H} \& \mathrm{E}$ (Yuanye Biotech) and observed under an IX 53 light microscope (Olympus) [20].

\section{Quantitative real-time polymerase chain reaction analysis}

Total RNA was extracted from 30 larvae at $12 \mathrm{~h}$ post-microinjection with RNAiso Plus. Then cDNA was synthesized following the manufacturer's instructions and the reverse transcription program was set as: $37^{\circ} \mathrm{C}$ for $15 \mathrm{~min}$ to start reaction, $85^{\circ} \mathrm{C}$ for $5 \mathrm{~s}$ to inactive enzymatic activity, $4^{\circ} \mathrm{C}$ to preserve. QRT-PCR was performed on a LightCycler 96 real-time PCR instrument (Roche) using TaKaRa Taq PCR kits. The amplification was performed at $95^{\circ} \mathrm{C}$ for $30 \mathrm{~s}$ followed by a total of 50 cycles at $95^{\circ} \mathrm{C}$ for $5 \mathrm{~s}, 60^{\circ} \mathrm{C}$ for $30 \mathrm{~s}$, and a final extension at $95^{\circ} \mathrm{C}$ for $5 \mathrm{~s}, 65^{\circ} \mathrm{C}$ for $60 \mathrm{~s}, 95^{\circ} \mathrm{C}$ for $1 \mathrm{~s}$. The gene expression ratios were calculated by the $2^{-\Delta \Delta \mathrm{ct}}$ method normalized to the expression level of $\beta$-actin. The primer sequences were as follows: $\beta$-actin, (forward) 5'-ATGGATGAGGAAATCGCTG-3' and (reverse) 5'-A TGCCAACCATCAC TCCC
TG-3'; IL-6, (forward) 5'-AGACCGCTGCCTGTCTAAAA-3' and (reverse) 5'-TTTGATG TCGTTCACCAGGA-3'; TNF- $\alpha$, (forward) $5{ }^{\prime}-$ GCTGGATCTTCAAAGTCGGGTGTA-3' and (reverse) 5'-TGTGAGT CTCAGCACACTTCCATC-3'; NF- $k B$, (forward) 5'-GAGCCCTTTGTG CAAGAGAC-3' and (reverse) 5'-TGGGATACGTCCTCCTGTTC-3'; I $\mathrm{B} \alpha \alpha$, (forward) 5'-GGTG GAAAGACTCCTGAAAGC-3' and (reverse) 5'-TGTAGTTAGGGAAGGTAAGAATG-3'; MyD88, (forward) 5'-GAGGATGGTGGTGGTCATCT-3' and (reverse) 5'-CGACAGGGATTAG CCGTTTA-3'; STAT3, (forward) 5'-CCCTGGGACTAACTC TGGCA-3' and (reverse) 5'-AGAG GTCCTGGATTGGCCTC-3'.

\section{Statistical analysis}

Data are presented as the mean \pm standard deviation (SD) from at least three independent experiments. Comparison of means among multiple groups was performed with one-way ANOVA using the statistical software GraphPad Prism 5.0. P $<0.05$ was considered statistically significant.

\section{Contributors' Statement}

Data collection: Y.R. Zheng, L.J. Deng, H. H. Cao, N. S. Xu, H. Y. Tian, B. J. Li, Z.B. Lu; design of the study: D. M. Zhang, W.C. Ye, L. Z. Yu, C. L. Fan, J. S. Liu; statistical analysis: Y. R. Zheng, L. J. Deng, H. H. Cao, N. S. Xu, D. M. Zhang, H. Y. Tian, B. J. Li, Z. B. Lu, W. C. Ye, L.Z. Yu, C. L. Fan, J.S. Liu; analysis and interpretation of the data: Y. R. Zheng, L.J. Deng, H. H. Cao, N. S. Xu, D. M. Zhang, H. Y. Tian, B.J. Li, Z.B. Lu, W.C. Ye, L.Z. Yu, C.L. Fan, J.S. Liu; drafting the manuscript: Y.R. Zheng, L.J. Deng, H.H. Cao, N.S. Xu, D.M. Zhang, H. Y. Tian, B. J. Li, Z. B. Lu, W. C. Ye, L.Z. Yu, C. L. Fan; critical revision of the manuscript: J.S. Liu.

\section{Acknowledgements}

This work was supported by the National Science Foundation of China (81973544, 81730110, 81903886), the Guangdong Province Universities and Colleges Pearl River Scholar Funded Scheme (GDHVPS2018), Young Elite Scientists Sponsorship Program by CACM (2019-QNRC2C14), the Guangzhou Education Bureau University Scientific Research project (201831845), and the Applied Basic Research Programs of Yunnan Province for Youths (No. 2017FD110).

\section{Conflict of Interest}

The authors declare that they have no conflict of interest.

\section{References}

[1] Zhang DM, Liu JS, Deng LJ, Chen MF, Yiu A, Cao HH, Tian HY, Fung KP, Kurihara H, Pan JX, Ye WC. Arenobufagin, a natural bufadienolide from toad venom, induces apoptosis and autophagy in human hepatocellular carcinoma cells through inhibition of PI3K/Akt/mTOR pathway. Carcinogenesis 2013; 34: 1331-1342

[2] Liu JS, Zhang DM, Li Y, Chen WM, Ruan ZX, Deng LJ, Wang LW, Tian HY, Yiu A, Fan CL, Luo H, Liu SW, Wang Y, Xiao GK, Chen LX, Ye WC. Discovery of bufadienolides as a novel class of ClC-3 chloride channel activators with antitumor activities. J Med Chem 2013; 56: 5734-5743

[3] Chen KK, Kovarikova A. Pharmacology and toxicology of toad venom. J Pharm Sci 1967; 56: 1535-1541 
[4] Qi J, Tan CK, Hashimi SM, Zulfiker AH, Good D, Wei MQ. Toad glandular secretions and skin extractions as anti-inflammatory and anticancer agents. Evid Based Complement Alternat Med 2014; 2014: 312684

[5] Chinese Pharmacopoeia Commission. Chinese Pharmacopoeia, 10th ed. Beijing: China Medical Science and Technology Press; 2015: 383-384

[6] Wen LL, Huang Y, Xie XB, Huang W, Yin JQ, Lin WQ, Jia Q, Zeng WA. Antiinflammatory and antinociceptive activities of bufalin in rodents. Mediators Inflamm 2014; 2014: 171839

[7] Chen YY, Lu HF, Hsu SC, Kuo CL, Chang SJ, Lin JJ, Wu PP, Liu JY, Lee CH, Chung JG, Chang JB. Bufalin inhibits migration and invasion in human hepatocellular carcinoma SK-Hep1 cells through the inhibitions of NF-kB and matrix metalloproteinase-2/-9-signaling pathways. Environ Toxicol 2015; 30: 74-82

[8] Xie SS, Spelmink L, Codemo M, Subramanian K, Pütsep K, HenriquesNormark B, Olliver M. Cinobufagin modulates human innate immune responses and triggers antibacterial activity. PLoS One 2016; 11: e0160734

[9] MacRae CA, Peterson RT. Zebrafish as tools for drug discovery. Nat Rev Drug Discov 2015; 14: 721-731

[10] Ganzen L, Venkatraman P, Pang CP, Leung YF, Zhang MZ. Utilizing zebrafish visual behaviors in drug screening for retinal degeneration. Int J Mol Sci 2017; 18: 1185

[11] Chua SC, Tan HQ, Engelberg D, Engelberg D, Lim L. Alternative experimental models for studying influenza proteins, host-virus interactions and anti-influenza drugs. Pharm 2019; 12: 147

[12] Trede NS, Langenau DM, Traver D, Look AT, Zon LI. The use of zebrafish to understand immunity. Immunity 2004; 20: 367-379

[13] Rojo I, de llárduya OM, Estonba A, Pardo MA. Innate immune gene expression in individual zebrafish after Listonella anguillarum inoculation. Fish Shellfish Immunol 2007; 23: 1285-1293

[14] Correa RG, Tergaonkar V, Ng JK, Dubova I, Izpisua-Belmonte JC, Verma IM. Characterization of NF-kappa B/I kappa B proteins in zebra fish and their involvement in notochord development. Mol Cell Biol 2004; 24: 5257-5268

[15] Rahman A, Fazal F. Blocking NF-KB: an inflammatory issue. Proc Am Thorac Soc 2011; 8: 497-503

[16] Guo D, Li JR, Wang Y, Lei LS, Yu CL, Chen NN. Cyclovirobuxinum D suppresses lipopolysaccharide-induced inflammatory responses in murine macrophages in vitro by blocking JAK-STAT signaling pathway. Acta Pharmacol Sin 2014; 35: 770-778

[17] Liu JS, Deng LJ, Tian HY, Ruan ZX, Cao HH, Ye WC, Zhang DM, Yu ZL. Antitumor effects and 3D-quantitative structure-activity relationship analysis of bufadienolides from toad venom. Fitoterapia 2019; 134: 362-371

[18] Renshaw S, Loynes C, Trushell D, Elworthy S, Ingham P, Whyte M. A transgenic zebrafish model of neutrophilic inflammation. Blood 2006; 108: 3976-3979

[19] Yang LL, Wang GQ, Yang LM, Huang ZB, Zhang WQ, Yu LZ. Endotoxin molecule lipopolysaccharide-induced zebrafish inflammation model: a novel screening method for anti-inflammatory drugs. Molecules 2014; 19: 2390-2409

[20] Gan L, Zheng YR, Deng L], Sun PH, Ye JX, Wei XD, Liu FF, Yu LZ, Ye WC, Fan CL, Liu JS, Zhang WQ. Diterpenoid lactones with anti-inflammatory effects from the aerial parts of Andrographis paniculate. Molecules 2019; 24: 2726

[21] Yang LL, Zhou XJ, Huang WJ, Fang Q, Hu JL, Yu LZ, Ma N, Zhang WQ. Protective effect of phillyrin on lethal LPS-induced neutrophil inflammation in zebrafish. Cell Physiol Biochem 2017; 43: 2074-2087

[22] Kim GR, Yang JY, Hwang KS, Kim SS, Chae JS, Kan H, Ahn JH, Lee WM, Ahn SH, Lee YM, Bae MA, Shin DS. Anti-inflammatory effect of a novel synthetic compound 1-((4-fluorophenyl)thio) isoquinoline in RAW264.7 macrophages and a zebrafish model. Fish Shellfish Immunol 2019; 87: $395-400$
[23] D’Alenon CA, Pea OA, Wittmann C, Gallardo VE, Jones RA, Loosli F, Liebel $\mathrm{U}$, Grabher C, Allende ML. A high-throughput chemically induced inflammation assay in zebrafish. BMC Biol 2010; 8: 151

[24] Li LL, Zhang S, Xin YF, Sun JY, Xie F, Yang L, Chen ZQ, Chen H, Liu F, Xuan YX. Role of Quzhou fructus Aurantii extract in preventing and treating acute lung injury and inflammation. Sci Rep 2018; 8: 1698

[25] Huang MY, Lin J, Lu K, Xu HG, Geng ZZ, Sun PH, Chen WM. Anti-inflammatory effects of cajaninstilbene acid and its derivatives. J Agric Food Chem 2016; 64: 2893-2900

[26] Fan HY, Gao ZF, Ji K, Li X, Wu JB, Liu Y, Wang XK, Liang HY, Liu YN, Li XT, Liu $P$, Chen DQ, Zhao $F$. The in vitro and in vivo anti-inflammatory effect of osthole, the major natural coumarin from Cnidium monnieri (L.) Cuss, via the blocking of the activation of the NF-KB and MAPK/p38 pathways. Phytomedicine 2019; 18: 1-11

[27] Varela M, Dios S, Novoa B, Figueras A. Characterisation, expression and ontogeny of interleukin-6 and its receptors in zebrafish (Danio rerio). Dev Comp Immunol 2012; 37: 97-106

[28] Kallen KJ. The role of transsignalling via the agonistic soluble IL-6 receptor in human diseases. Biochim Biophys Acta 2002; 1592: 323-343

[29] Cheng BC, Ma XQ, Kwan HY, Tse KW, Cao HH, Su T, Shu X, Wu ZZ, Yu ZL. An herbal formula consisting of Rosae Multiflorae Fructus and Lonicerae Japonicae Flos inhibits inflammatory mediators in LPS-stimulated RAW 264.7 macrophages. J Ethnopharmacol 2014; 153: 922-927

[30] O'Neill LA, Bowie AG. The family of five: TIR-domain-containing adaptors in Toll-like receptor signaling. Nat Rev Immunol 2007; 7: 353-364

[31] Kim SY, Park SM, Hwanqbo M, Lee JR, Byun SH, Ku SK, Cho IJ, Kim SC, Jee SY, Park SJ. Cheongsangbangpung-tang ameliorated the acute inflammatory response via the inhibition of NF-kappaB activation and MAPK phosphorylation. BMC Complement Altern Med 2017; 17: 46

[32] Torraca V, Mostowy S. Zebrafish infection: from pathogenesis to cell biology. Trends Cell Biol 2018; 28: 143-156

[33] Mortaz E, Alipoor SD, Adcock IM, Mumby S, Koenderman L. Update on neutrophil function in severe inflammation. Front Immunol 2018; 9: 2171

[34] Zhou HL, Cao HH, Zheng YR, Lu ZB, Chen YY, Liu DY, Yang HY, Quan JY, Huo CY, Liu JS, Yu LZ. Liang-Ge-San, a classic traditional Chinese medicine formula, attenuates acute inflammation in zebrafish and RAW 264.7 cells. J Ethnopharmacol 2020; 249: 112427

[35] Pharmacopoeia Committee of People's Republic China. Pharmacopoeia of People's Republic China. Beijing: Chemical Industry Publishers; 2015: 383-384

[36] Zhan X, Wu H, Wu H, Wang R, Luo C, Gao B, Chen ZW, Li QL. Metabolites from Bufo gargarizans (Cantor, 1842): A review of traditional uses, pharmacological activity, toxicity and quality control. J Ethnopharmacol 2020; 246: 112178

[37] Li J], Zhang Y, Han LW, Tian QP, He QX, Wang XM, Sun C, Hai J, Liu KC. Tenacissoside $\mathrm{H}$ exerts an anti-inflammatory effect by regulating the $\mathrm{nf}-\kappa \mathrm{b}$ and p38 pathways in zebrafish. Fish Shellfish Immunol 2018; 83: 205-212

[38] Xiong ST, Wu J], Jing J, Huang PP, Li Z, Mei J, Gui JF. Loss of stat3 function leads to spine malformation and immune disorder in zebrafish. Sci Bull 2017; 62: 185-196

[39] He G, Karin M. NF-KB and STAT3- key players in liver inflammation and cancer. Cell Res 2011; 21: 159-168

[40] Grivennikov SI, Karin M. Dangerous liaisons: STAT3 and NF-kB collaboration and crosstalk in cancer. Cytokine Growth Factor Rev 2010; 21: $11-$ 19

[41] Westerfield M. The Zebrafish Book. A Guide for the laboratory Use of Zebrafish (Danio rerio). Eugene: University of Oregon Press; 2000 